\title{
Anti-protozoal activity of aporphine and protoberberine alkaloids from Annickia kummeriae (Engl. \& Diels) Setten \& Maas (Annonaceae)
}

\author{
Hamisi M Malebo ${ }^{1 *}$, Tanja Wenzler ${ }^{2}$, Monical Cal ${ }^{2}$, Sauda M Swaleh ${ }^{3}$, Maurice O Omolo ${ }^{7}$, Ahmed Hassanali ${ }^{4}$,
} Urs Séquin ${ }^{5}$, Daniel Häussinger ${ }^{5}$, Petur Dalsgaard ${ }^{6}$, Matthias Hamburger ${ }^{6}$, Reto Brun ${ }^{2}$ and Isaiah 0 Ndiege $^{3}$

\begin{abstract}
Background: Malaria, trypanosomiasis and leishmaniasis have an overwhelming impact in the poorest countries in the world due to their prevalence, virulence and drug resistance ability. Currently, there is inadequate armory of drugs for the treatment of malaria, trypanosomiasis and leishmaniasis. This underscores the continuing need for the discovery and development of new anti-protozoal drugs. Consequently, there is an urgent need for research aimed at the discovery and development of new effective and safe anti-plasmodial, anti-trypanosomal and anti-leishmanial drugs.

Methods: Bioassay-guided chromatographic fractionation was employed for the isolation and purification of antiprotozoal alkaloids.

Results: The methanol extract from the leaves of Annickia kummeriae from Tanzania exhibited a strong anti-plasmodial activity against the multi-drug resistant Plasmodium falciparum $\mathrm{K} 1$ strain $\left(\mathrm{IC}_{50} 0.12 \pm 0.01 \mathrm{\mu g} / \mathrm{ml}\right.$, selectivity index (SI) of 250, moderate activity against Trypanosoma brucei rhodesiense STIB 900 strain (IC $502.50 \pm 0.19 \mu \mathrm{g} / \mathrm{ml}, \mathrm{SI} 12)$ and mild activity against Leishmania donovani axenic MHOM-ET-67/82 strain (IC $509.25 \pm 0.54$ Mg/ml, SI 3.2). Bioassay-guided chromatographic fractionation led to the isolation of four pure alkaloids, lysicamine (1), trivalvone (2), palmatine (3), jatrorrhizine (4) and two sets of mixtures of jatrorrhizine (4) with columbamine (5) and palmatine (3) with (-)-tetrahydropalmatine (6). The alkaloids showed low cytotoxicity activity $\left(C_{50} 30\right.$ - >90 $\left.\mathrm{mg} / \mathrm{ml}\right)$, strong to moderate anti-plasmodial activity $\left(\mathrm{IC}_{50} 0.08 \pm 0.001-2.4 \pm 0.642 \mu \mathrm{g} / \mathrm{ml}, \mathrm{SI} 1.5-1,154\right)$, moderate to weak anti-trypanosomal $\left(\mathrm{IC}_{50} 2.80 \pm 0.001-14.3 \pm 0.001 \mu \mathrm{g} / \mathrm{ml}, \mathrm{SI} 2.3-28.1\right)$ and anti-leishmanial activity IC $502.7 \pm 0.001-20.4 \pm 0.003 \mu \mathrm{g} / \mathrm{ml}$, SI 1.7-15.6)
\end{abstract}

Conclusion: The strong anti-plasmodial activity makes these alkaloids good lead structures for drug development programs.

Keywords: Annickia kummeriae, Enantia kummeriae, Annonaceae, Alkaloids, Aporphine, Protoberberine, Antiplasmodial, Antitrypanosomal, Antileishmanial, Cytotoxicity

\section{Background}

Protozoal diseases such as malaria, trypanosomiasis and leishmaniasis have an overwhelming impact in the poorest countries in the world [1]. Due to their prevalence, virulence and drug resistance, they are the most serious and widespread parasitic diseases in the tropics [1-5]. The inadequate armory of drugs for the treatment

\footnotetext{
* Correspondence: Malebo@hotmail.com

${ }^{1}$ Department of Traditional Medicine Research, National Institute for Medical Research, P.O. Box 9653, Dar es Salaam, Tanzania

Full list of author information is available at the end of the article
}

of malaria, trypanosomiasis and leishmaniasis; and the high cost of new drugs coupled with the rapid development of resistance to new anti-parasitic drugs are some of the limiting factors in the fight against these tropical diseases. This underscores the continuing need for the discovery and development of new anti-protozoal drugs. Consequently, there is an urgent need for research aimed at the discovery and development of new effective and safe anti-plasmodial, anti-trypanosomal and antileishmanial drugs. In view of the complicated situations in dealing with parasitic infections, chemotherapy 
remains a dependable strategy in disease control. In the development of new drugs, the plant kingdom is considered to be important source for lead compounds owing to the successful use in traditional treatment of various ailments since antiquity [6]. Historically, medicinal plants have served as sources of new pharmaceutical products like quinine and artemisinin [7] and inexpensive starting materials for the synthesis of many known drugs. Research focused on the identification of medicinal natural products from higher plants for the discovery of new parasitic agents has been ongoing for more than five decades.

Ethnomedical information revealed that several Annickia (formerly Enantia) species are used widely for the treatment of malaria and other ailments [8]. Enantia chlorantha and E. polycarpa are used traditionally in the treatment of malaria and fever in West and Central Africa [9,10]. Consequently, previous pharmacological investigations on genus Enantia revealed promising anti-protozoal activity with the stem-bark extract of E. chlorantha showing strong in vitro anti-plasmodial activity against $P$. falciparum $\mathrm{K} 1$ strain $\left(\mathrm{IC}_{50} 0.126 \mu \mathrm{g} / \mathrm{ml}\right)$ and good selectivity (SI 616) [11]. Furthermore, E. chlorantha aqueous and ethanolic extracts exhibited in vivo activity with $\mathrm{ED}_{50}$ values of $6.9 \mathrm{mg}$ g- 1 and $0.34 \mathrm{mg}$ g-1, respectively, against Plasmodium yoelii in experimentally infected mice [12]. The chemistry of E. chlorantha and E. polycarpa has been extensively studied [10,13-15]. Several quinoline and isoquinoline alkaloids including protoberberines, quinine and dihydroquinidine have been isolated from E. polycarpa [16,17]. Protoberberine alkaloids have been identified as the major anti-protozoal alkaloids in E. chlorantha and E. polycarpa [16-19]. Protoberberines isolated from Enantia chlorantha exhibited significant antiplasmodial activity against both CQ-sensitive and resistant strains of $P$. falciparum: palmatine (3) ( $\mathrm{IC}_{50} 0.27$ and $0.16 \mu \mathrm{g} / \mathrm{ml}$, respectively) and jatrorrhizine (4) $\left(\mathrm{IC}_{50} 4.2\right.$ and $1.61 \mu \mathrm{g} / \mathrm{ml}$, respectively) in vitro [18]. A mixture of protoberberine alkaloids from Enantia chlorantha containing; palmatine (3), jatrorrhizine (4) and columbamine (5) (Hepasor), were shown to prevent liver injury from chemically induced traumatization and also promoted the healing process after the injury [20] in experimental mice. Palmatine (3) and jatrorrhizine (4) demonstrated to inhibit the growth of Babesia gibsoni at concentrations ranging from 100 and $10 \mu \mathrm{g} / \mathrm{ml}$ [21]. In an effort to identify the molecular basis of activity, we undertook bioassay-guided fractionation of extracts of Annickia kummeriae (Engl. \& Diels) Setten \& Maas (formerly, Enantia kummeriae), a plant traditionally used for the treatment of malaria in Tanzania. Bioassay-guided chromatography led to the isolation of lysicamine (1), trivalvone (2), palmatine (3), jatrorrhizine (4) and two sets of mixtures of jatrorrhizine (4) with columbamine (5) and palmatine (3) with (-)-tetrahydropalmatine (6) as shown in Figure 1.

\section{Methods}

\section{General procedures}

Analytical grade and double-distilled solvents were used for the extraction and chromatographic isolation and purification of compounds. Analytical thin layer chromatography (TLC) was performed on both aluminium and plastic sheets precoated with silica gel $60 \mathrm{~F}_{254}$ (Merck) with a $0.2 \mathrm{~mm}$ layer thickness. Visualisation of TLC spots was carried out under UV light at 254 or $366 \mathrm{~nm}$ and by spraying with Dragendorff reagent. Preparative thin layer chromatography (PTLC) was done using normal phase silica gel $60 \mathrm{~F}_{254}$ (Merck) precoated on glass plates $(20 \times 20 \mathrm{~cm})$, with varying thickness $(0.5,1.0$ or $2.0 \mathrm{~mm})$. Detection was done under UV light at 254 or $366 \mathrm{~nm}$. Preparative high speed counter-current chromatograph (HSCCC) was done on Potomac (P.C. Inc., Buffalo, NYUSA) equipped with three preparative multilayer coils (wound with $1.7 \mathrm{~mm}$ internal diameter, polytetrafluoroethylene PTFE tubing of $80 \mathrm{ml}$ and $240 \mathrm{ml}$ connected in series with a total capacity of $320 \mathrm{ml}$ ) run at a revolution speed of $611 \mathrm{rpm}$ and the solvent was pumped into the column with a Büchi B-688 chromatography pump. Continuous monitoring of the effluent was achieved with a Model UV-II detector Monitor at $254 \mathrm{~nm}$. A manual sample injection valve with a $20 \mathrm{~mL}$ loop was used to introduce the sample into the column and the eluent collected in a Büchi B-684 fraction collector. Melting points of recrystallized solids were measured on a Büchi B-540 apparatus and are uncorrected. IR spectra were measured on a Perkin Elmer model 1600 FT-IR spectrophotometer using potassium bromide pellets. Mass spectra were measured on mass spectrometer VG 70S (EIMS) and a Finnigan MAT 312 FABMS. NMR spectra were measured on Bruker Avance $400\left({ }^{1} \mathrm{H}\right.$ NMR $400 \mathrm{MHz} ;{ }^{13} \mathrm{C}$ NMR $101 \mathrm{MHz})$, Bruker VRX $500\left({ }^{1} \mathrm{H}\right.$ NMR $500 \mathrm{MHz} ;{ }^{13} \mathrm{C}$ NMR $125 \mathrm{MHz})$ and Bruker DRX $600\left({ }^{1} \mathrm{H}\right.$ NMR $600 \mathrm{MHz} ;{ }^{13} \mathrm{C}$ NMR $\left.150.9 \mathrm{MHz}\right)$. The purity level was determined by LC-MS (Agilent 1100 system equipped with an Agilent 1100 DAD MS detector; column Nucleodur $\mathrm{C}_{18}, 5 \mu \mathrm{m}, 125 \mathrm{~mm} \times 4.0 \mathrm{~mm}$ internal diameter (i.d); mobile phase A: $0.01 \%$ aqueous formic acid and mobile phase B: acetonitrile). The structures were assigned by NMR and mass spectrometry. The isolated compounds were screened for anti-plasmodial, anti-trypanosomal, anti-leishmanial and cytotoxic activity.

\section{Plant materials and chemicals}

Plant materials were collected at Amani Nature Reserve (Tanzania) in August 2003 and identified at the Department of Botany, University of Nairobi (Kenya) where the voucher specimen (HM 2004/04) is deposited in the 


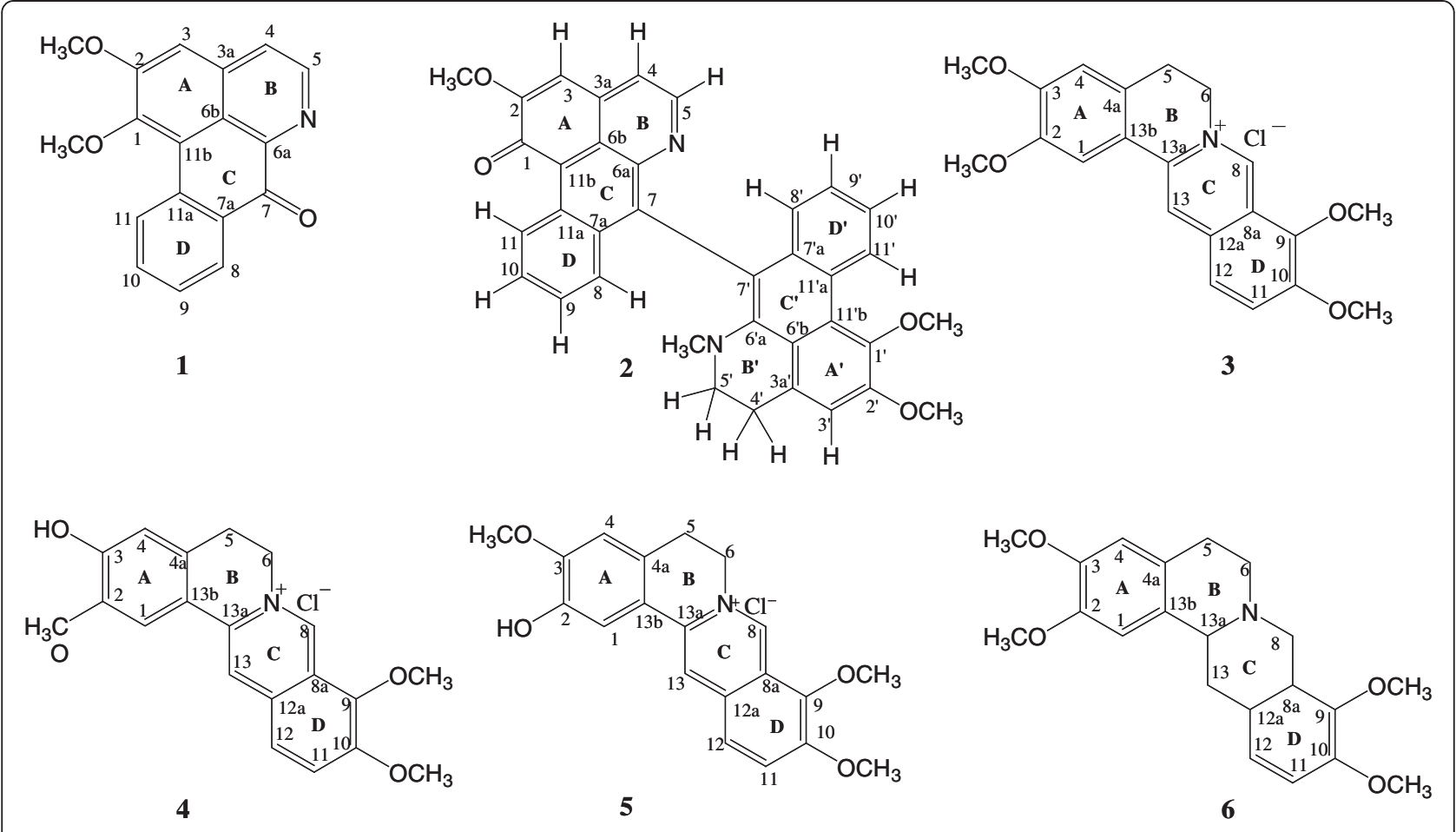

Figure 1 Chemical structures of isolated compounds.

Herbarium. The plant materials (leaves, root-bark and stem-bark) were dried under shade for 14 days and ground to powder. The ground air-dried Annickia kummeriae leaves, stem and root bark (1.12, 1.55 and $1.77 \mathrm{~kg}$, respectively) were extracted sequentially, at room temperature for 48 hours with intermittent shaking, with petroleum ether (PE), dichloromethane (DCM) and methanol $(\mathrm{MeOH})$. The extract was filtered off, the solvent removed under reduced pressure at $30^{\circ} \mathrm{C}$, dried further under a stream of nitrogen for 24 hours before being weighed and used for biological assays.

Chemicals used were: Formic acid, hydrochloric acid, sulphuric acid, acetic acid, citric acid, $p$-anisaldehyde, vanillin, dragendorf reagent, sodium chloride, sodium hydrogen carbonate, acetone, $n$-hexane, petroleum ether, dichloromethane, chloroform, ethyl acetate, toluene, ethanol and methanol were also bought from Kobian Chemicals, Nairobi, Kenya and Fluka AG in Switzerland. Analytical grade or double-distilled solvents were used for the extraction and chromatographic isolation and purification of compounds. $\left[{ }^{3} \mathrm{H}\right]$-Hypoxanthine and Rosewell Park Memorial Institute 1640 (RPMI 1640) powdered medium were bought from Gibco Laboratories, California, U.S.A whereas, dextrose, Giemsa stain, resazurin dye, glycerol and $N$-2-hydroxyethylpiperazine $N$-2-ethanesulfonic acid (HEPES) were bought from Sigma-Aldrich, Germany. Deuterated solvents: chloroform and methanol used for spectroscopic analysis were bought from Fluka AG, Switzerland.

\section{Bioassay of extracts and guided isolation of aporphine and protoberberine alkaloids In vitro anti-plasmodial assay}

Anti-plasmodial activity was evaluated against the multidrug resistant Plasmodium falciparum K1 strain (resistant to chloroquine and pyrimethamine), using the parasite cultivation method of Trager and Jensen, 1976 [22] and the assay originally described by Desjardins et al., 1979 [23] with slight modifications by Matile \& Pink [24].

\section{In vitro anti-trypanosomal assay}

The in vitro anti-trypanosomal activity was evaluated against Trypanosoma brucei rhodesiense STIB 900 strain, using the cultivation method of Baltz et al.,1985 [25] whereby the Minimum Essential Medium (MEM) was supplemented with $0.2 \mathrm{mM}$ 2-mercaptoethanol, $1 \mathrm{mM}$ sodium pyruvate, $0.5 \mathrm{mM}$ hypoxanthine and $15 \%$ heatinactivated horse serum. The assay was performed according to Räz et al., 1997 [26].

\section{In vitro anti-leishmanial assay}

The in vitro anti-leishmanial assay was carried out against axenic amastigote forms of Leishmania donovani MHOM-ET-67/82 strain according to the procedure described by Ganapaty et al., 2006 [27]. 


\section{Cytotoxicity assay}

The in vitro cytotoxicity assay was carried out using rat skeletal myoblast (L-6) cells according to the procedure described by Ganapaty et al., 2006 [27]. Cytotoxicity activity of the test extract and compounds $\left(\mathrm{IC}_{50}\right)$ was compared with cytotoxicity activity of the standard cytotoxic compound and used to calculate selectivity index. Selectivity indices (SI) were calculated using the formula:

$$
\mathrm{SI}=\frac{\text { Cytotoxicity of standard } \operatorname{drug}\left(\mathrm{CC}_{50}\right)}{\text { Cytotoxicity of test extract } / \text { compound }\left(\mathrm{IC}_{50}\right)}
$$

\section{Bioassay guided isolation of antiplasmodial compounds}

The ground air-dried leaves, stem bark and root bark of Annickia kummeriae (1.12 kg, $1.55 \mathrm{~kg}$ and $1.77 \mathrm{~kg}$, respectively) was extracted sequentially with solvents of increasing polarity (petroleum ether, dichloromethane and methanol) for 48 hours at room temperature. The resulting extracts were obtained by filtration and concentration in vacuo at $30^{\circ} \mathrm{C}$. After screening for anti-plasmodial, anti-trypanosomal, anti-leishmanial and cytotoxic activity, the crude methanolic leaf extract, which was the most active, was selected for bioassay-guided fractionation and isolation of anti-protozoal compounds. The methanolic leaf extract (3 g) was fractionated using HSCCC through stepwise elution with a biphasic solvent

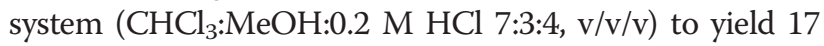
fractions which were screened for anti-plasmodial and cytotoxic activity. The HSCCC fractions AKLM 4-AKLM 6 and AKLM 7-AKLM 10, which exhibited antiplasmodial activity, were combined based on similarity of the TLC profile. Repeated HSCCC of fraction AKLM 2 using stepwise elution with a biphasic solvent system $\left(\mathrm{CHCl}_{3}: \mathrm{MeOH}: 0.2 \mathrm{M} \mathrm{HCl}\right.$ 7:3:4) gave 11 sub-fractions (AKLM 1-AKLM 11) which were screened for antiplasmodial and cytotoxic activity. Column chromatography of sub-fractions AKLM 2.10 and AKLM 2.11 on silica gel $(0.040-0.063 \mathrm{~mm})$ eluting with $\mathrm{C}_{6} \mathrm{H}_{14}$-EtOAc 1:1, and EtOAc- $\mathrm{MeOH}$ 8:2 followed by purification with sephadex LH-20 eluting with $\mathrm{MeOH}-\mathrm{CHCl}_{3}$ 1:1 and preparative TLC (PTLC) on silica gel $\mathrm{PF}_{254}$ with $\mathrm{CHCl}_{3}$ : $\mathrm{MeOH}: \mathrm{HCO}_{2} \mathrm{H}$ 98:1.8:0.2 yielded (10.21 mg) of lysicamine (1) ( $0.01 \%$ yield, $92 \%$ purity) and $(8.10 \mathrm{mg})$ of trivalvone (2) $(0.01 \%$ yield:, $95 \%$ purity), respectively. Repeated HSCCC eluting with $\mathrm{CHCl}_{3}: \mathrm{MeOH}: 0.2 \mathrm{M} \mathrm{HCl} 7: 3: 4$ of the combined fractions AKLM 7-AKLM 10 gave 20 subfractions (AKLM 7.1-AKLM 7.20). TLC analysis indicated a similar pure compound in AKLM 7.6-AKLM 7.13 which was recrystallized from methanol to yield $(1.52 \mathrm{~g})$ of palmatine (3) $(1.84 \%$ yield, $91 \%$ purity). Column chromatography (silica gel $0.040-0.063 \mathrm{~mm}$ ) of subfractions AKLM 7.15-AKLM 7.16, with similar TLC profiles, eluting sequentially with $\mathrm{CHCl}_{3}: \mathrm{MeOH}: \mathrm{HCO}_{2} \mathrm{H}$ 9:0.75:0.25, 8:1.75:0.25, 6:3.75:0.5 and 5:4.5:0.5 followed by recrystallization from methanol yielded $(40.82 \mathrm{mg})$ of jatrorrhizine (4) $(0.05 \%$ yield, $94 \%$ purity $)$. Repeated HSCCC of of the combined fractions AKLM 4-AKLM 6 with $\mathrm{CHCl}_{3}: \mathrm{MeOH}: 0.2 \mathrm{M} \mathrm{HCl}$ 7:3:4 gave 16 sub-fractions (AKLM 4.1-AKLM 4.16). Column chromatography (silica gel 0.040-0.063 mm) with $\mathrm{MeOH} ; \mathrm{CH}_{2} \mathrm{Cl}_{2}: \mathrm{HCO}_{2} \mathrm{H}$ 4:15:1 followed by PTLC (silica gel $\mathrm{PF}_{254}$ ) with $\mathrm{MeOH}-\mathrm{CH}_{2} \mathrm{Cl}_{2}$ $\mathrm{HCO}_{2} \mathrm{H}$ 5:14:1 gave (34.2) of an inseparable mixture (1.2:1.0) of jatrorrhizine (4) and columbamine (5) $(0.04 \%$ yield). Similarly, $\mathrm{HSCCC}$ of of AKLM 16 with $\mathrm{CHCl}_{3}$ : $\mathrm{MeOH}: 0.2 \mathrm{M} \mathrm{HCl}$ 7:3:4 gave 12 sub-fractions (AKLM 16.1-AKLM 16.12). Column chromatography (silica gel $0.040-0.063 \mathrm{~mm}$ ) of the combined sub-fractions AKLM 16.8-AKLM 16.10 with $\mathrm{MeOH} ; \mathrm{CH}_{2} \mathrm{Cl}_{2} ; \mathrm{HCO}_{2} \mathrm{H}$ 5:14:1 followed by PTLC (silica gel $\mathrm{PF}_{254}$ ) with $\mathrm{MeOH}: \mathrm{CH}_{2} \mathrm{Cl}_{2}$ $\mathrm{HCO}_{2} \mathrm{H}$ 4:15:1 yielded (28.2 mg) of an inseparable mixture (1.1:1.0) of palmatine (3) and (-)-tetrahydropalmatine (6) ( $0.03 \%$ yield).

\section{Structural elucidation of isolated compounds}

The chemical structures of isolated compounds were established on the basis of spectroscopical data as Infrared (IR), $1 \mathrm{D}\left({ }^{1} \mathrm{H},{ }^{13} \mathrm{C}, \mathrm{DEPT}\right.$ 135) and 2D-NMR experiments; Heteronuclear Multiple-Quantum Correlation (HMQC), correlation spectroscopy (COSY) and Heteronuclear Multiple Bond Correlation (HMBC) plus Mass Spectroscopy (MS) data. The ${ }^{13} \mathrm{C}$ NMR data were assigned with the help of HMQC and DEPT 135 experiments while, the connectivity's of the molecular fragments were established by HMBC, COSY and NOESY. The analysis of the spectra and structure elucidation was also facilitated by comparison of observed and published ${ }^{1} \mathrm{H}$ and ${ }^{13} \mathrm{C}$ NMR data for the compounds.

Lysicamine (1): yellow needles (10.21 mg), m.p. $209-211^{\circ} \mathrm{C}$, ${ }^{1} \mathrm{H}$ NMR $\left(\mathrm{CDCl}_{3}, 600 \mathrm{MHz}\right) \delta 7.57(1 \mathrm{H}, \mathrm{s}, \mathrm{H}-3), 8.07(1 \mathrm{H}$, d, $J=5.2 \mathrm{~Hz}, \mathrm{H}-4), 8.77(1 \mathrm{H}, \mathrm{d}, J=5.2 \mathrm{~Hz}, \mathrm{H}-5), 8.48(1 \mathrm{H}$, dd, $J=9.0,1.8 \mathrm{~Hz}, \mathrm{H}-8), 7.63(1 \mathrm{H}, \mathrm{t}, J=9.0,1.2 \mathrm{~Hz}, \mathrm{H}-9)$, $7.86(1 \mathrm{H}, \mathrm{t}, J=9.0,1.4 \mathrm{~Hz}, \mathrm{H}-10), 9.26(1 \mathrm{H}, \mathrm{dd}, J=9.0$, $1.2 \mathrm{~Hz}, \mathrm{H}-11), 4.13\left(3 \mathrm{H}, \mathrm{s}, 1-\mathrm{OCH}_{3}\right), 4.06\left(3 \mathrm{H}, \mathrm{s}, 2-\mathrm{OCH}_{3}\right)$. ${ }^{13} \mathrm{C} \mathrm{NMR}\left(\mathrm{CDCl}_{3}, 600 \mathrm{MHz}\right) \delta 145.3$ (s, C-1), 158.2 (s, C-2), 108.3 (d, C-3), 157.5 (s, C-3a), 125.8 (s, C-4), 145.0 (d, C-5), 155.6 (s, C-6a), 123.3 (s, C-6b), 182.6 (s, C-7), 132.8 (s, C-7a), 129.3 (d, C-8), 129.8 (d, C-9), 135.7 (d, C-10), 129.7 (s, C-11), 135.8 (s, 11a), 120.0 (s, 11b), 60.1 (q, 1- $\left.\mathrm{OCH}_{3}\right), 56.4$ (q, 1- $\mathrm{OCH}_{3}$ ). MS: m/z 291 (100\%), 275 (15\%), 248 (84\%), 233 (9\%), 188 (4\%), 177 (12\%). The molecular mass of $\mathbf{1}$ is $\mathrm{m} / \mathrm{z} 291 \mathrm{amu}$ which is consistent with the formula $\mathrm{C}_{18} \mathrm{H}_{13} \mathrm{NO}_{3}$. All the data for compound 1 were consistent with the reported values for lysicamine, which was first isolated from Lysichiton camtschatcense (Araceae) [28,29]. Lysicamine (1) has been widely isolated from several plant species [30] however; this is 
the first report on the presence of lysicamine (1) from A. kummeriae (Annonaceae).

Trivalvone (2): brown crystals $(8.10 \mathrm{mg})$, m.p. 256$\left.258^{\circ} \mathrm{C}\right),{ }^{1} \mathrm{H}$ NMR $\left(\mathrm{CDCl}_{3}, 500 \mathrm{MHz}\right) \delta 6.87(1 \mathrm{H}, \mathrm{s}, \mathrm{H}-3)$, $7.54(1 \mathrm{H}, \mathrm{d}, J=4.1, \mathrm{H}-4), 8.90(1 \mathrm{H}, \mathrm{d}, J=4.1, \mathrm{H}-5), 7.76$ $(1 \mathrm{H}, \mathrm{d}, J=9.0,2.1, \mathrm{H}-8), 7.35(1 \mathrm{H}, \mathrm{t}, J=9.0,2.1, \mathrm{H}-9)$, $7.85(1 \mathrm{H}, \mathrm{t}, J=9.0,2.1, \mathrm{H}-10), 10.20(1 \mathrm{H}, \mathrm{d}, J=9.0$, $2.1 \mathrm{H}-11), 7.18\left(1 \mathrm{H}, \mathrm{s}, \mathrm{H}-3^{\prime}\right), 2.95\left(2 \mathrm{H}, \mathrm{m}, \mathrm{H}-4^{\prime}\right), 3.26$ $\left(2 \mathrm{H}, \mathrm{m}, \mathrm{H}-5^{\prime}\right), 6.70\left(1 \mathrm{H}, \mathrm{d}, \mathrm{H}-8^{\prime}\right), 7.12\left(1 \mathrm{H}, \mathrm{t}, \mathrm{H}-9^{\prime}\right)$, $7.43\left(1 \mathrm{H}, \mathrm{t}, \mathrm{H}-10^{\prime}\right), 9.75\left(1 \mathrm{H}, \mathrm{d}, \mathrm{H}-11^{\prime}\right), 4.07(3 \mathrm{H}, \mathrm{s}, 2-$ $\left.\mathrm{OCH}_{3}\right), 4.01\left(3 \mathrm{H}, \mathrm{s}, 1^{\prime}-\mathrm{OCH}_{3}\right), 4.07\left(3 \mathrm{H}, \mathrm{s}, 2^{\prime}-\mathrm{OCH}_{3}\right), 2.15$ $\left(3 \mathrm{H}, \mathrm{s}, 1^{\prime}-\mathrm{N}-\mathrm{CH}_{3}\right) .{ }^{13} \mathrm{C}$ NMR $\left(\mathrm{CDCl}_{3}, 500 \mathrm{MHz}\right) \delta 181.0$ (s, C-1), 151.3 (s, C-2), 107.5 (d, C-3), 127.9 (d, C-3a), 127.9 (d, C-4), 151.0 (d, C-5), 156.6 (s, C-6a), 122.6 (s, C-6b), 134.0 (s, C-7), 142.5 (s, C-7a), 132.6 (d, C-8), 128.7 (d, C-9), 127.0 (d, C-10), 121.9 (d, C-11), 122.7 (s, C-11a), 136.2 (s, C-11b), 145.9 (s, C-1'), 150.6 (s, C-2'), 112.8 (d, C-3'), 130.9 (s, C-3' a), 25.6 (t, C-4'), 49.7 (t, C-5' ), 143.8 (s, C-6' a), 121.1 (s, C-6' b), 122.4 (s, C-7'), 134.4 (s, C-7' a), 127.9 (d, C-8'), 126.7 (d, C-9'), 126.6 (d, C-10'), 124.7 (d, C-11'), 126.2 (s, C-11' a), 127.4 (s, C-11' b), 56.3 (q, 2- $\left.-\mathrm{OCH}_{3}\right), 60.0\left(\mathrm{q}, 1^{\prime}-\mathrm{OCH}_{3}\right), 56.6\left(\mathrm{q}, 2^{\prime}-\mathrm{OCH}_{3}\right), 41.6$ $\left(\mathrm{q}, \mathrm{N}-\mathrm{CH}_{3}\right)$. MS: m/z $554\left([\mathrm{M}+2]^{+}, 90.4 \%\right), 553\left([\mathrm{M}+1]^{+}\right.$, 41.3\%), $292(\mathrm{M} / 2+\mathrm{H}, 8.4 \%)$.

The molecular mass of 2 is $m / z 552 \mathrm{amu}$, which is consistent with the formula $\mathrm{C}_{36} \mathrm{H}_{28} \mathrm{~N}_{2} \mathrm{O}_{4}$. The absence of any fragmentation in the region $\mathrm{m} / \mathrm{z}$ 552-292 suggested a dimeric structure for 2 , resulting from a $\mathrm{C}-7 \rightarrow \mathrm{C}^{-} \mathbf{7}^{\prime}$ oxidative coupling between the two aporphine units [31]. The NMR and MS data confirmed the structure of the bis-aporphine alkaloid, trivalvone (2), a rare alkaloid first reported in 1990 from Trivalvaria macrophylla (Annonaceae) [31] and subsequently from Piptostigma fugax (Annonaceae) [32]. This is the first report on the presence of trivalvone (2) from Annickia kummeriae (Annonaceae).

Palmatine (3): yellow solid (1.52 g), m.p. $203-205^{\circ} \mathrm{C}$, ${ }^{1} \mathrm{H}$ NMR $\left(\mathrm{CD}_{3} \mathrm{OD}, 500 \mathrm{MHz}\right) \delta 7.63(1 \mathrm{H}, \mathrm{s}, \mathrm{H}-1), 7.04$ $(1 \mathrm{H}, \mathrm{s}, \mathrm{H}-4), 3.30(2 \mathrm{H}, \mathrm{t}, J=6.3, \mathrm{H}-5), 4.95(2 \mathrm{H}, \mathrm{t}, J=6.3$, H-6), $9.75(1 \mathrm{H}, \mathrm{br}, \mathrm{s}, \mathrm{H}-8), 8.09(1 \mathrm{H}, \mathrm{d}, J=9.1, \mathrm{H}-11)$, $8.01(1 \mathrm{H}, \mathrm{d}, J=9.1, \mathrm{H}-12), 8.79(1 \mathrm{H}, \mathrm{s}, \mathrm{H}-13), 3.94(3 \mathrm{H}$, $\left.\mathrm{s}, 2-\mathrm{OCH}_{3}\right), 4.00\left(3 \mathrm{H}, \mathrm{s}, 3-\mathrm{OCH}_{3}\right), 4.22\left(3 \mathrm{H}, \mathrm{s}, 9-\mathrm{OCH}_{3}\right)$, $4.10\left(3 \mathrm{H}, \mathrm{s}, 10-\mathrm{OCH}_{3}\right) .{ }^{13} \mathrm{C} \mathrm{NMR}\left(\mathrm{CD}_{3} \mathrm{OD}, 500 \mathrm{MHz}\right) \delta$ 110.4 (d, C-1), 151.3 (s, C-2), 154.2 (s, C-3), 112.7 (d, C-4), 130.4 (s, C-4a), 28.2 (t, C-5), 56.4 (t, C-6), 146.7 (d, C-8), 123.6 (s, C-8a), 146.1 (s, C-9), 152.3 (d, C-10), 128.4 (d, C-11), 124.9 (d, C-12), 135.6 (s, C-12a), 121.7 (d, C-13), 140.1 (s, C-13a), 120.8 (s, C-13b), 57.5 (q, 2- $\left.-\mathrm{OCH}_{3}\right), 57.1$ (q, 3- $\left.-\mathrm{OCH}_{3}\right), 63.0$ (q, 9- $\mathrm{OCH}_{3}$ ), 57.8 (q, 10- $\left.\mathrm{OCH}_{3}\right)$. MS: m/z 352 (75\%), 337 (6\%), 336 (7\%), 308 (20\%), 154 (100\%), 77 (25\%), 39 (20\%). The mass spectrum of 3 exhibited a molecular ion peak at $m / z 352$, which is consistent with the formula $\mathrm{C}_{21} \mathrm{H}_{22} \mathrm{NO}_{4}^{+}$(D.B.E 11.5). The non-integer value of the index of hydrogen deficiency suggested that $\mathbf{3}$ could be a quaternary ammonium salt consistent with palmatine (3). All the observed data for $\mathbf{3}$ were consistent with the reported values for palmatine except for the interchange of $\mathrm{H}-11$ and $\mathrm{H}-12$ in ${ }^{1} \mathrm{H}$ NMR [33,34]. Palmatine (3) has been previously reported from many plant families: Papaveraceae, Berberidaceae, Fumariaceae, Menispermaceae, Ranunculaceae, Rutaceae, Annonaceae, Magnoliaceae and Convolvulaceae [35].

Jatrorrhizine (4): orange crystals (40.82 mg), m.p. 204-206 ${ }^{\circ} \mathrm{C},{ }^{1} \mathrm{H}$ NMR $\left(\mathrm{CDCl}_{3}, 600 \mathrm{MHz}\right) \delta 7.57(1 \mathrm{H}, \mathrm{s}$, $\mathrm{H}-1), 6.76(1 \mathrm{H}, \mathrm{s}, \mathrm{H}-4), 3.17(2 \mathrm{H}, \mathrm{t}, J=6.1 \mathrm{~Hz}, \mathrm{H}-5), 4.87$ $(2 \mathrm{H}, \mathrm{t}, J=6.1 \mathrm{~Hz}, \mathrm{H}-6), 9.67(1 \mathrm{H}, \mathrm{t}, \mathrm{br}, \mathrm{s}, \mathrm{H}-8), 8.07(1 \mathrm{H}, \mathrm{d}$, $J=9.1 \mathrm{~Hz}, \mathrm{H}-11), 7.96(1 \mathrm{H}, \mathrm{d}, J=9.1 \mathrm{~Hz}, \mathrm{H}-12), 8.68(1 \mathrm{H}$, s, $\mathrm{H}-13), 3.99\left(3 \mathrm{H}, \mathrm{s}, 2-\mathrm{OCH}_{3}\right), 4.19\left(3 \mathrm{H}, \mathrm{s}, 9-\mathrm{OCH}_{3}\right), 4.10$ $\left(3 \mathrm{H}, \mathrm{s}, 10-\mathrm{OCH}_{3}\right) .{ }^{13} \mathrm{C} \mathrm{NMR}\left(\mathrm{CDCl}_{3}, 600 \mathrm{MHz}\right) \delta 109.6$ (d, C-1), 150.9 (s, C-2), 152.1 (s, C-3), 116.6 (d, C-4), 130.5 (s, C-4a), 27.8 (t, C-5), 57.4 (t, C-6), 145.7 (d, C-8), 122.9 (s, C-8a), 145.9 (s, C-9), 151.5 (s, C-10), 128.1 (d, C-11), 124.2 (d, C-12), 135.7 (s, C12a), 120.2 (d, C-13), 141.1 (s, C-13a), 117.1 (s, C-13b), 56.7 (q, 2- $\left.-\mathrm{OCH}_{3}\right), 62.4$ (q, 9- $\mathrm{OCH}_{3}$ ), 57.7 (q, 10- $\mathrm{OCH}_{3}$ ). MS: m/z 338 (28\%), 176 (55\%), 154 (100\%), 77 (29\%), 41 (25\%). MS exhibited a molecular ion peak at $m / z 338$ consistent with the formula $\mathrm{C}_{20} \mathrm{H}_{20} \mathrm{NO}_{4}^{+}$D.B.E of 11.5 indicating presence of a quaternary ammonium salt. All the data for compound $\mathbf{4}$ were consistent with the reported values for jatrorrhizine [35]. Jatrorrhizine (4) has been previously reported from several plant families: Papaveraceae, Berberidaceae, Fumariaceae, Menispermaceae, Ranunculaceae, Rutaceae, Annonaceae, Magnoliaceae and Convolvulaceae [35].

Columbamine (5): orange solid (34.2 mg, mp. 235$\left.240^{\circ} \mathrm{C}\right),{ }^{1} \mathrm{H}$ NMR $\left(\mathrm{CDCl}_{3}, 600 \mathrm{MHz}\right) \delta 7.51(1 \mathrm{H}, \mathrm{s}, \mathrm{H}-1)$, $7.00(1 \mathrm{H}, \mathrm{s}, \mathrm{H}-4), 3.24(2 \mathrm{H}, \mathrm{t}, J=6.0 \mathrm{~Hz}, \mathrm{H}-5), 4.92$ $(2 \mathrm{H}, \mathrm{t}, J=6.0 \mathrm{~Hz}, \mathrm{H}-6), 9.74(1 \mathrm{H}, \mathrm{t}, \mathrm{br}, \mathrm{s}, \mathrm{H}-8), 8.10(1 \mathrm{H}, \mathrm{d}$, $J=9.0 \mathrm{~Hz}, \mathrm{H}-11), 7.99(1 \mathrm{H}, \mathrm{d}, J=9.0 \mathrm{~Hz}, \mathrm{H}-12), 8.63(1 \mathrm{H}$, s, $\mathrm{H}-13), 3.95\left(3 \mathrm{H}, \mathrm{s}, 2-\mathrm{OCH}_{3}\right), 4.20\left(3 \mathrm{H}, \mathrm{s}, 9-\mathrm{OCH}_{3}\right), 4.10$ $\left(3 \mathrm{H}, \mathrm{s}, 10-\mathrm{OCH}_{3}\right) \cdot{ }^{13} \mathrm{C} \mathrm{NMR}\left(\mathrm{CDCl}_{3}, 600 \mathrm{MHz}\right) \delta 109.2$ (d, C-1), 149.2 (s, C-2), 152.8 (s, C-3), 111.7 (d, C-4), 127.8 (s, C-4a), 27.7 (t, C-5), 57.4 (t, C-6), 146.1 (d, C-8), 123.2 (s, C-8a), 145.5 (s, C-9), 151.7 (s, C-10), 127.8 (d, C-11), 124.3 (d, C-12), 135.2 (s, C12a), 120.8 (d, C-13), 140.0 (s, C-13a), 120.5 (s, C-13b), $57.5\left(\mathrm{q}, 2-\mathrm{OCH}_{3}\right), 62.4$ (q, 9- $\left.\mathrm{OCH}_{3}\right), 56.4\left(\mathrm{q}, 10-\mathrm{OCH}_{3}\right) . \mathrm{MS}: \mathrm{m} / \mathrm{z} 338(28 \%)$, $176(55 \%), 154(100 \%), 77(29 \%), 41(25 \%)$. The MS of columbamine (5) exhibited a molecular ion peak at $\mathrm{m} / \mathrm{z}$ 338 consistent with the formula $\mathrm{C}_{20} \mathrm{H}_{20} \mathrm{NO}_{4}^{+}$(D.B.E 11.5) confirming the presence of quaternary nitrogen atom. All the data were consistent with the reported values for columbamine (5) [35]. Columbamine (5) has been previously reported from several plant families: Papaveraceae, Berberidaceae, Fumariaceae, Menispermaceae, Ranunculaceae, Rutaceae, Annonaceae, Magnoliaceae and Convolvulaceae [35]. 
(-)-Tetrahydropalmatine (6): yellow amorphous solid (28.2 mg, m.p. 204-205 $\left.{ }^{\circ} \mathrm{C}\right),{ }^{1} \mathrm{H}$ NMR $(600 \mathrm{MHz}$, $\left.\mathrm{CD}_{3} \mathrm{OD}\right) \delta 6.89(1 \mathrm{H}, \mathrm{s}, \mathrm{H}-1), 6.90(1 \mathrm{H}, \mathrm{s}, \mathrm{H}-4), 3.28(1 \mathrm{H}$, $\left.\mathrm{m}, \mathrm{H}-5_{e q}\right), 3.33\left(1 \mathrm{H}, \mathrm{m}, \mathrm{H}-5_{a x}\right), 3.60\left(1 \mathrm{H}, \mathrm{m}, \mathrm{H}-6_{e q}\right), 3.84$ $\left(1 \mathrm{H}, \mathrm{m}, \mathrm{H}-6_{a x}\right), 4.91\left(1 \mathrm{H}, \mathrm{d}, J=15.7, \mathrm{H}-8_{e q}\right), 4.78(1 \mathrm{H}, \mathrm{d}$, $\left.J=15.7, \mathrm{H}-8_{a x}\right), 7.07(1 \mathrm{H}, \mathrm{d}, J=8.5, \mathrm{H}-11), 6.98(1 \mathrm{H}$, d, $J=8.5, \mathrm{H}-12), 3.15$ ( $\left.1 \mathrm{H}, \mathrm{dd}, J=18,10.3, \mathrm{H}-13_{a x}\right), 3.50$ $(1 \mathrm{H}, \mathrm{dd}, J=18,5.7, \mathrm{H}-13$ eq $), 4.76(1 \mathrm{H}, \mathrm{dd}, J=10.3,5.7$, $\mathrm{H}-13 \mathrm{a}), 3.84\left(3 \mathrm{H}, \mathrm{s}, 2-\mathrm{OCH}_{3}\right), 3.85\left(3 \mathrm{H}, \mathrm{s}, 3-\mathrm{OCH}_{3}\right), 3.90$ $\left(3 \mathrm{H}, \mathrm{s}, 9-\mathrm{OCH}_{3}\right), 3.87\left(3 \mathrm{H}, \mathrm{s}, 10-\mathrm{OCH}_{3}\right),{ }^{13} \mathrm{C} \mathrm{NMR}$ $\left(600 \mathrm{MHz}, \mathrm{CD}_{3} \mathrm{OD}\right) \delta 111.3$ (d, C-1), 151.6 (s, C-2), 150.4 (s, C-3), 115.5 (d, C-4), 125.7 (s, C-4a), 24.6 (t, C-5), 53.3 (t, C-6), 61.4 (d, C-8), 121.4 (s, C-8a), 147.1 (s, C-9), 153.1 (s, C-10), 115.0 (d, C-11), 125.0 (d, C-12), 123.7 (s, C12a), 35.4 (d, C-13), 67.7 (s, C-13a), 125.7 (s, C-13b), 53.5 (q, 2- $\left.-\mathrm{OCH}_{3}\right), 56.4\left(\mathrm{q}, 3-\mathrm{OCH}_{3}\right), 63.1\left(\mathrm{q}, 9-\mathrm{OCH}_{3}\right), 56.1$ (q, $\left.10-\mathrm{OCH}_{3}\right)$. The MS of (-)-tetrahydropalmatine (6) exhibited molecular ion peak at $\mathrm{m} / z 356$ consistent with the formulae $\mathrm{C}_{21} \mathrm{H}_{27} \mathrm{NO}_{4}$ (D.B.E 10). The odd molecular mass confirmed the presence of a neutral alkaloid. Comparison of the observed spectral data with literature values for (-)-tetrahydropalmatine (6) [35]. (-)-Tetrahydropalma tine (6) has been previously reported from several plant families: Papaveraceae, Berberidaceae, Fumariaceae, Menispermaceae, Ranunculaceae, Rutaceae, Annonaceae, Magnoliaceae and Convolvulaceae [35]. This is the first report on the presence of (-)-tetrahydropalmatine (6) from Annickia kummeriae (Annonaceae).

\section{Results and discussion}

The in vitro anti-plasmodial, anti-trypanosomal, antileishmanial and lower cytotoxicity activity of extracts from $A$. kummeriae were previously published elsewhere [36]. Results of the fractionation of methanolic extract of A. kummeriae leaves by HSCCC are shown in Table 1. Of the 17 fractions 8 (47.1\%) exhibited very strong antiplasmodial activity against $P$. falciparum $\mathrm{K} 1$ strain $\left(\mathrm{IC}_{50}\right.$ $0.05 \pm 0.01-0.13 \pm 0.02 \mu \mathrm{g} / \mathrm{ml}$ ) with excellent selectivity (SI >692), $3(17.6 \%)$ showed strong activity $\left(\mathrm{IC}_{50} 0.45 \pm\right.$ $0.15-0.87 \pm 0.1 \mu \mathrm{g} / \mathrm{ml}$ ) with satisfactory selectivity (SI 22.9-145.2) while the remaining 6 (35.3\%) exhibited moderate activity $\left(\mathrm{IC}_{50} 1.0 \pm 0.22-5.0 \pm 0.31 \mu \mathrm{g} / \mathrm{ml}\right)$ with moderate selectivity (SI 18.0-90.0). The antiplasmodial activity $\left(\mathrm{IC}_{50}\right)$ and cytotoxicity $\left(\mathrm{CC}_{50}\right)$ of the 17 HSCCC fractions were compared with the standard drugs: chloroquine, artemisinin and podophyllotoxin.

Fractions AKLM 9-12 $\left(\mathrm{IC}_{50} 0.05 \pm 0.01-0.09 \pm 0.04 \mu \mathrm{g} / \mathrm{ml}\right.$, SI 1,000.0-1,800.0) were of particular interest since the antiplasmodial activity compared very well to $C Q$, and was only 30-fold lower than that of artemisinin, and is not cytotoxic. Others with promising anti-plasmodial activity included: AKLM $8(0.09 \pm 0.004 \mu \mathrm{g} / \mathrm{ml}$, SI $>1,000$, 1.4 and 45 fold lower than CQ and artemisinin, respectively), AKLM 16 ( $\mathrm{IC}_{50} 0.09 \pm 0.03 \mu \mathrm{g} / \mathrm{ml}$, SI 933.3, 1.4 and
45 fold lower than $\mathrm{CQ}$ and artemisinin, respectively), AKLM $7\left(\mathrm{IC}_{50} 0.11 \pm 0.02 \mu \mathrm{g} / \mathrm{ml}\right.$, SI >818.2; 1.7 and 55 fold lower than CQ and artemisinin, respectively), AKLM 15 $\left(\mathrm{IC}_{50} 0.13 \pm 0.02 \mu \mathrm{g} / \mathrm{ml}, \mathrm{SI}>692,2.1\right.$ and 65 fold lower than CQ and artemisinin, respectively) and AKLM 6 (IC 50 $0.45 \pm 0.15 \mu \mathrm{g} / \mathrm{ml}$, SI 131.1, 7.1 and 225 fold lower than CQ and artemisinin, respectively) all of which were not toxic. HSCCC re-fractionation of AKLM $2\left(\mathrm{IC}_{50} 0.87 \pm\right.$ $0.1 \mu \mathrm{g} / \mathrm{ml}$, SI 22.9) gave 11 sub-fractions but only two (AKLM 2.10 and AKLM 2.11 with $\mathrm{IC}_{50} 0.64 \pm 0.34$ and $0.89 \pm 0.20 \mu \mathrm{g} / \mathrm{ml}$, respectively) showed moderate antiplasmodial activity against $P$. falciparum $\mathrm{K} 1$ strain as the mother fraction: (Table 2).

In fact the remaining 9 sub-fractions exhibited lower antiplasmodial activity $\left(\mathrm{IC}_{50} 1.09 \pm 0.26-5.0 \pm 0.53 \mu \mathrm{g} / \mathrm{ml}\right.$ ) and poor to satisfactory selectivity (SI 11.9-72.6) than the mother fraction. In the comparison of the antiplasmodial activity $\left(\mathrm{IC}_{50}\right)$, HSCCC fraction AKLM 2.10 showed 13.9 and 445 fold lower activity than CQ and artemisinin, respectively) whereas, AKLM 2.11 revealed 10 and 320 fold lower activity than CQ and artemisinin, respectively). Both fractions were not cytotoxic. The methanolic leaf extract of A. kummeriae gave four pure alkaloids: lysicamine (1), trivalvone (2), palmatine (3), jatrorrhizine (4), and a pair of inseparable mixtures of two alkaloids each: jatrorrhizine (4)/columbamine (5), and palmatine (3)/(-)-tetrahydropalmatine (6), which were tested for anti-plasmodial, anti-trypanosomal, anti-leishmanial and cytotoxic activity.

Bioassay guided HSCCC fractionation of AKLM 2, using $P$. falciparum $\mathrm{K} 1$ strain, led to two major alkaloids lysicamine (1), an aporphine alkaloid and trivalvone (2), a bis-aporphine alkaloid as confirmed by spectral analysis. Lysicamine (1) has been widely isolated from several plant species [30]. However, this is the first report on its isolation from A. kummeriae (Annonaceae). Trivalvone (2) is a rare bis-aporphine alkaloid first reported in 1990 from Trivalvaria macrophylla (Annonaceae) [31] and subsequently from Piptostigma fugax (Annonaceae) [32]. This is also the first report on its presence in $A$. kummeriae (Annonaceae).

Similarly, bioassay-led HSCCC fractionation of the combined antiplasmodial fractions AKLM 7-AKLM 10, led to the isolation of two protoberberine alkaloids, which were confirmed by spectral analysis as palmatine (3) $[10,33-35,37]$ and jatrorrhizine (4) [34,35,37]. Likewise, bioassay-directed HSCCC fractionation of the combined anti-plasmodial fractions AKLM 4-AKLM 6 yielded an inseparable mixture (1.2:1.0) of protoberberine alkaloids, which were confirmed as jatrorrhizine (4) [34,35] and columbamine (5) [35,38,39] by spectral analysis. Bioassay-informed HSCCC fractionation of the anti-plasmodial fraction AKLM 16 gave an inseparable mixture (1.1:1.0) of protoberberine 
Table 1 Anti-plasmodial activity $\left(\mathrm{IC}_{50}\right)$ and cytotoxicity $\left(\mathrm{CC}_{50}\right)$ of HSCCC fractions of Annickia kummeriae methanolic leaf extract

\begin{tabular}{|c|c|c|c|c|c|c|c|}
\hline Fraction & Wt (mg) & $\begin{array}{r}\text { P. falciparum K1 } \\
I_{50}(\mu \mathrm{g} / \mathrm{ml}) \\
\end{array}$ & $\begin{array}{r}\text { Cytotoxicity } \mathrm{CC}_{50} \\
(\mu \mathrm{g} / \mathrm{ml})\end{array}$ & SI & $\begin{array}{l}I C_{50} \mathrm{fr} . \\
I C_{50} \mathrm{CQ}\end{array}$ & $\begin{array}{r}I C_{50} \mathrm{fr} . \\
I C_{50} \text { Art }\end{array}$ & $\begin{array}{r}I C_{50} \mathrm{fr} . \\
I C_{50} \mathrm{Pdx} \\
\end{array}$ \\
\hline AKLM & 15,000 & $0.12 \pm 0.01$ & $30.0 \pm 0.8$ & 250 & 1.9 & 60 & 3,333 \\
\hline AKLM1 & 562 & $5.0 \pm 0.31$ & $>90.0$ & $>18$ & 79.4 & 2500 & $>10,000$ \\
\hline AKLM2 & 2,999 & $0.87 \pm 0.1$ & $20.0 \pm 3.3$ & 23 & 13.8 & 435 & 2,222 \\
\hline AKLM3 & 1,534 & $3.01 \pm 0.81$ & $78.0 \pm 5.4$ & 26 & 47.8 & 1,505 & 8,667 \\
\hline AKLM 4 & 157 & $1.34 \pm 0.33$ & $76.0 \pm 1.44$ & 57 & 21.3 & 670 & 8,444 \\
\hline AKLM 5 & 249 & $3.6 \pm 0.2$ & $>90.0$ & $>25$ & 57.1 & 1,800 & $>10,000$ \\
\hline AKLM 6 & 427 & $0.45 \pm 0.15$ & $59.0 \pm 1.5$ & 131 & 7.1 & 225 & 6,556 \\
\hline AKLM 7 & 165 & $0.11 \pm 0.02$ & $>90.0$ & $>818$ & 1.7 & 55 & $>10,000$ \\
\hline AKLM 8 & 221 & $0.09 \pm 0.04$ & $>90.0$ & $>1,000$ & 1.4 & 45 & $>10,000$ \\
\hline AKLM 9 & 680 & $0.06 \pm 0.02$ & $>90.0$ & $>1,500$ & 1.0 & 30 & $>10,000$ \\
\hline AKLM 10 & 1,295 & $0.05 \pm 0.02$ & $>90.0$ & $>1,800$ & 0.8 & 25 & $>10,000$ \\
\hline AKLM 11 & 1,679 & $0.05 \pm 0.01$ & $>90.0$ & $>1,800$ & 0.8 & 25 & $>10,000$ \\
\hline AKLM 12 & 1,056 & $0.06 \pm 0.03$ & $>90.0$ & $>1,500$ & 1.0 & 30 & $>10,000$ \\
\hline AKLM 13 & 878 & $0.62 \pm 0.4$ & $>90.0$ & $>145$ & 9.8 & 310 & $>10,000$ \\
\hline AKLM 14 & 948 & $1.0 \pm 0.22$ & $>90.0$ & $>90$ & 15.9 & 500 & $>10,000$ \\
\hline AKLM 15 & 1,232 & $0.13 \pm 0.02$ & $>90.0$ & $>692$ & 2.1 & 65 & $>10,000$ \\
\hline AKLM 16 & 416 & $0.09 \pm 0.03$ & $84.0 \pm 3.91$ & 933 & 1.4 & 45 & 9,333 \\
\hline AKLM 17 & 498 & $1.67 \pm 0.43$ & $>90.0$ & $>54$ & 26.5 & 835 & $>10,000$ \\
\hline
\end{tabular}

Fr. - HSCCC fraction of Annickia kummeriae leaves methanolic extract, P. falciparum K1 used for anti-plasmodial assays, rat myoblast L- 6 cells used for cytotoxicity assays, $C Q$ chloroquine $\left(\mathrm{IC}_{50} 0.063 \pm 0.034 \mu \mathrm{g} / \mathrm{ml}\right)$, Art artemisinin $\left(\mathrm{IC}_{50} 0.002 \pm 0.00001 \mu \mathrm{g} / \mathrm{ml}\right), P d x$ podophyllotoxin $(\mathrm{IC} 50.009 \pm 0.003 \mu \mathrm{g} / \mathrm{ml})$.

alkaloids, which upon spectral analysis were confirmed a palmatine (3) and (-)-tetrahydropalmatine (6) $[33-35,40,41]$. This is the first report on the presence of columbamine (5) and (-)-tetrahydropalmatine (6) in A. kummeriae.
The four pure alkaloids, lysicamine (1), trivalvone (2), palmatine (3), jatrorrhizine (4) and the two sets of mixtures of jatrorrhizine (4) with columbamine (5) and palmatine (3) with (-)-tetrahydropalmatine (6) were found to exhibit in vitro anti-plasmodial activity against

Table 2 Anti-plasmodial activity ( $\left.\mathrm{IC}_{50}\right)$ and cytotoxicity $\left(\mathrm{CC}_{50}\right)$ data of sub-fractions of fraction 2 of Annickia kummeriae methanolic leaf extract

\begin{tabular}{|c|c|c|c|c|c|c|c|}
\hline Fraction & Weight (mg) & $\begin{array}{r}\text { P. falciparum K1 } \\
\mathrm{IC}_{50}(\mu \mathrm{g} / \mathrm{ml})\end{array}$ & $\begin{array}{l}\text { Cytotoxicity } \\
\mathrm{CC}_{50}(\mu \mathrm{g} / \mathrm{ml})\end{array}$ & SI & $\begin{array}{l}I C_{50} \mathrm{fr} . \\
I C_{50} C Q\end{array}$ & $\begin{array}{l}I C_{50} \mathrm{fr} . \\
I C_{50} \text { Art }\end{array}$ & $\begin{array}{l}C C_{50} \mathrm{fr} . \\
C C_{50} \mathrm{Pdx}\end{array}$ \\
\hline AKLM 2 & 2,500 & $0.90 \pm 0.11$ & $21.0 \pm 3.87$ & 23.3 & 14 & 450 & 3,500 \\
\hline AKLM 2.1 & 15.3 & $5.0 \pm 1.31$ & $>90$ & 18.0 & 78 & 2,500 & $>15,000$ \\
\hline AKLM 2.2 & 26.9 & $1.09 \pm 0.26$ & $79.1 \pm 7.60$ & 72.6 & 17 & 545 & 13,183 \\
\hline AKLM 2.3 & 57.6 & $4.11 \pm 0.29$ & $53.1 \pm 9.20$ & 12.9 & 64 & 2,055 & 8,850 \\
\hline AKLM 2.4 & 98.7 & $1.16 \pm 0.18$ & $13.8 \pm 1.80$ & 11.9 & 18 & 580 & 2,300 \\
\hline AKLM 2.5 & 126.1 & $3.63 \pm 0.04$ & $24.6 \pm 3.30$ & 6.8 & 57 & 1,815 & 4,100 \\
\hline AKLM 2.6 & 115.7 & $1.23 \pm 0.31$ & $39.5 \pm 2.20$ & 32.1 & 19 & 615 & 6,583 \\
\hline AKLM 2.7 & 239.0 & $3.41 \pm 0.48$ & $85.4 \pm 4.60$ & 25.0 & 53 & 1,705 & 14,233 \\
\hline AKLM 2.8 & 478.3 & $5.0 \pm 0.53$ & $>90$ & 18.0 & 78 & 2,500 & $>15,000$ \\
\hline AKLM 2.9 & 351.8 & $2.40 \pm 0.57$ & $56.7 \pm 8.00$ & 23.6 & 38 & 1,200 & 9,450 \\
\hline AKLM 2.10 & 301.9 & $0.89 \pm 0.20$ & $35.3 \pm 5.31$ & 39.7 & 14 & 445 & 5,883 \\
\hline AKLM 2.11 & 672.8 & $0.64 \pm 0.34$ & $44.7 \pm 4.45$ & 69.8 & 10 & 320 & 7,450 \\
\hline
\end{tabular}

HSCCC High speed counter current chromatography; AKLM Annickia kummeriae leaf methanol extract, $P$. falciparum K1 used for anti-plasmodial assays, rat myoblast L-6 cells used for cytotoxicity assays, CQ chloroquine (IC $\left.{ }_{50} 0.063 \pm 0.034 \mu \mathrm{g} / \mathrm{ml}\right)$, Art - artemisinin $(\mathrm{IC} 50.002 \pm 0.00001 \mu \mathrm{g} / \mathrm{ml})$, Pdx - podophyllotoxin $\left(\mathrm{IC}_{50} 0.009 \pm 0.003 \mu \mathrm{g} / \mathrm{ml}\right)$. 
Table 3 Anti-protozoal activity ( $\left.\mathrm{IC}_{50}\right)$ and cytotoxicity $\left(\mathrm{CC}_{50}\right)$ data of alkaloids from Annickia kummeriae

\begin{tabular}{|c|c|c|c|c|c|c|c|}
\hline \multirow[b]{2}{*}{ Compound } & \multicolumn{2}{|c|}{ P. falciparum $\mathrm{K} 1$} & \multicolumn{2}{|c|}{ T.b. rhodesiense } & \multicolumn{2}{|l|}{ L. donovani } & \multirow{2}{*}{$\begin{array}{c}\mathrm{L}-6 \text { cells } \\
\mathrm{CC}_{50} \pm \mathrm{S} . \mathrm{E}(\mu \mathrm{g} / \mathrm{ml})\end{array}$} \\
\hline & $\mathrm{IC}_{50} \pm \mathrm{S} . \mathrm{E}(\mu \mathrm{g} / \mathrm{ml})$ & $\mathrm{SI}$ & $\mathrm{IC}_{50} \pm \mathrm{S} . \mathrm{E}(\mu \mathrm{g} / \mathrm{ml})$ & $\overline{\mathrm{SI}}$ & $\mathrm{IC}_{50} \pm \mathrm{S} . \mathrm{E}(\mu \mathrm{g} / \mathrm{ml})$ & SI & \\
\hline Lysicamine (1) & $2.4 \pm 0.642$ & 1.5 & $3.7 \pm 0.001$ & 2.3 & $2.7 \pm 0.001$ & 1.7 & $1.6 \pm 0.01$ \\
\hline Trivalvone (2) & $1.6 \pm 0.232$ & 28.3 & $14.3 \pm 0.001$ & 3.2 & $2.9 \pm 0.001$ & 15.6 & $45.3 \pm 0.02$ \\
\hline Palmatine (3) & $0.080 \pm 0.001$ & 1,154 & $3.2 \pm 0.004$ & 28.1 & $7.8 \pm 0.001$ & 11.5 & $>90$ \\
\hline Jatrorrhizine (4) & $0.24 \pm 0.002$ & 375.0 & $4.2 \pm 0.002$ & 21.4 & $20.4 \pm 0.03$ & 4.4 & $>90$ \\
\hline Jatrorrhizine (4)/columbamine (5) & $0.14 \pm 0.017$ & 358.6 & $4.0 \pm 0.001$ & 12.6 & $13.1 \pm 0.02$ & 3.8 & $50.2 \pm 0.08$ \\
\hline Palmatine (3)/tetrahydro- palmatine (6) & $0.098 \pm 0.002$ & 629.6 & $4.3 \pm 0.005$ & 14.4 & $7.0 \pm 0.06$ & 8.81 & $61.7 \pm 0.01$ \\
\hline
\end{tabular}

P. falciparum - K1 strain, T. b. rhodesiense - STIB 900 strain, L. donovani - MHOM-ET-67/L82, L-6 - rat skeletal myoblast cells, IC 50 - inhibitory concentration for 50\% of tested parasites, $\mathrm{CC}_{50}$ - cytotoxic concentration for $50 \%$ of tested cells, chloroquine $\mathrm{IC}_{50} 0.063 \pm 0.03$, artemisinin $\mathrm{IC}_{50} 0.002 \pm 0.0001$, melarsoprol IC $\mathrm{C}_{50} 0.002 \pm$ 0.0001 , miltefosine $I_{50} 0.11 \pm 0.001$, podophyllotoxin $I C_{50} 0.009 \pm 0.0003$.

the multi-drug resistant $P$. falciparum K1 strain, antitrypanosomal activity against the $T . b$. rhodesiense STIB 900 and anti-leishmanial activity against $L$. donovani axenic MHOM-ET-67/82 strain (Table 3).

Four protoberberine alkaloids showed strong in vitro activity against $P$. falciparum $\mathrm{K} 1$ strain $\left(\mathrm{IC}_{50} 0.08 \pm 0.001\right.$ $0.24 \pm 0.002 \mu \mathrm{g} / \mathrm{ml}$ ) singly and as mixtures and good selectivity $(\mathrm{SI}>375$ ) while the remaining two aporphine alkaloids exhibited moderate anti-plasmodial activity $\left(\mathrm{IC}_{50}\right.$ $1.6 \pm 0.23-2.4 \pm 0.04 \mu \mathrm{g} / \mathrm{ml})$ singly and poor to moderate selectivity (SI 1.6-28.8). Palmatine (3) exhibited the strongest anti-plasmodial activity against $P$. falciparum K1 strain $\left(\mathrm{IC}_{50} 0.08 \pm 0.001 \mu \mathrm{g} / \mathrm{ml}\right)$ and a good selectivity (SI 1,154). Jatrorrhizine (4) also showed strong antiplasmodial activity $(0.24 \pm 0.002 \mu \mathrm{g} / \mathrm{ml})$ and good selectisity (SI >375). Protoberberine alkaloids were of particular interest as they showed strong anti-plasmodial activity which was very close to that of chloroquine as shown in Table 4. Our data indicate that, palmatine (3) and jatrorrhizine (4) with other protoberberine alkaloids such as columbamine (5) and (-)-tetrahydropalmatine (6) are active constituents responsible for the antiplasmodial activity of A. kummeriae. However, the protoberberines and the monomeric aporphine alkaloids were only moderately active against $T$. $b$. rhodesiense STIB 900 strain in vitro $\left(\mathrm{IC}_{50} 2.8 \pm 0.001-4.3 \pm 0.0005 \mu \mathrm{g} / \mathrm{ml}\right)$ with moderate selectivity (SI 14.4-28.1) whereas the bis-aporphine alkaloid, trivalvone (2) was inactive $\left(\mathrm{IC}_{50} 14.3 \pm 0.001 \mu \mathrm{g} / \mathrm{ml}\right)$. Similarly, the two aporphine alkaloids showed moderate activity against $L$. donovani MHOM-ET-67/L82 axenic amastigotes in vitro: lysicamine (1) $\left(\mathrm{IC}_{50} 2.7 \pm 0.0001 \mu \mathrm{g} / \mathrm{ml}\right)$ with no selectivity (SI 1.5) and trivalvone (2) ( $\mathrm{IC}_{50} 2.9 \pm$ $0.0001 \mu \mathrm{g} / \mathrm{ml}$ ) with moderate selectivity (SI 15.6) while the remaining four protoberberine alkaloids were inactive $\left(\mathrm{IC}_{50}\right.$ $7.0 \pm 0.001-20.4 \pm 0.001 \mu \mathrm{g} / \mathrm{ml})$. Moderate to mild antileishmanial activity (23.6-185.5 folds) was noted for all the isolated compounds compared to miltefosine $\left(\mathrm{IC}_{50} 0.11 \pm\right.$ $0.001 \mu \mathrm{g} / \mathrm{ml}$ ) as shown in Table 4 .

The literature indicate that plants that contain protoberberine and aporphine alkaloids are used in folkloric medicine as anti-hypertensive, anti-cancer, antiseptic, sedatives, analgesics, anti-inflammatory, antifungal, anti-bacterial and anti-protozoal [21,40]. The in vitro anti-plasmodial activity of protoberberine alkaloids has been previously reported. However, none of them has been shown to be active in vivo [16-19,35]. Oxygenation at C-2, C-3 (ring A) and C-9, C-10 (ring D) together with the presence of quaternary nitrogen atom in position 7 in protoberberine alkaloids have already been identified as the structural motifs required for strong antiplasmodial activity [42]. The relationship between the oxygenation and the antiplasmodial activity provides clues for possible molecular frameworks for synthesis and structure-activity relationship studies

Table 4 Comparison of anti-protozoal activity $\left(\mathrm{IC}_{50}\right)$ and cytotoxicity $\left(\mathrm{CC}_{50}\right)$ of alkaloids from Annickia kummeriae with standard drugs

\begin{tabular}{|c|c|c|c|c|c|}
\hline Compound & $\mathrm{IC}_{50} \mathrm{cpd} I \mathrm{IC}_{50} \mathrm{CQ}$ & 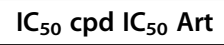 & $I C_{50} \mathrm{cpd} \mathrm{IC} C_{50} \mathrm{Mel}$ & $I C_{50}$ cpd IC $C_{50}$ Milt & $\mathrm{CC}_{50} \mathrm{cpd} \mathrm{CC_{50 } \mathrm { Pdx }}$ \\
\hline Lysicamine (1) & 38.1 & $1,200.0$ & $1,850.0$ & 23.6 & 177.8 \\
\hline Trivalvone (2) & 25.4 & 800.0 & $7,150.0$ & 26.4 & $5,033.3$ \\
\hline Palmatine (3) & 1.3 & 40.0 & $1,600.0$ & 70.9 & $>10,000$ \\
\hline Jatrorrhizine (4) & 3.8 & 120 & $2,100.0$ & 185.5 & $>10,000$ \\
\hline Jatrorrhizine (4) + columbamine (5) (1.2:1.0) & 2.2 & 69.0 & $2,000.0$ & 119.1 & $5,577.8$ \\
\hline $\begin{array}{l}\text { Palmatine (3) + (-)-tetrahydropalmatine (6) } \\
(1.1: 1.0)\end{array}$ & 1.6 & 49.0 & $2,150.0$ & 63.64 & $6,855.6$ \\
\hline
\end{tabular}

Cpd isolated compound, CQ chloroquine, Art artemisinin, Mel melarsoprol, Pdx podophyllotoxin. 
which might lead to the identification of pharmacophore(s) for new generation of isoquinoline antiplasmodial drug(s).

\section{Conclusion}

To the best of our knowledge, this is the first report on the anti-plasmodial and anti-leishmanial activity of A. kummeriae, in vitro anti-trypanosomal activity of palmatine (3); anti-plasmodial, anti-trypanosomal, anti-leishmanial and cytotoxicity activity of trivalvone (2); anti-leishmanial and anti-trypanosomal activity of jatrorrhizine (4) and of the two sets of mixtures: jatrorrhizine (4)/columbamine (5) (1.2:1.0) and palmatine (3)/(-)-tetrahydropalmatine (6) (1.1:1.0). The present phytochemical and pharmacological results indicate that $A$. kummeriae, a traditional remedy for malaria and fever, exhibits a wide array of biological activities, which could be attributed to the constituent aporphine and protoberberine alkaloids. The protoberberine alkaloids exhibit good antiprotozoal activity in vitro and comparably low cytotoxicity. In contrast, the activity and selectivity of aporphine alkaloids is moderate. Given the reported lack of in vivo activity of protoberberine alkaloids, further investigations should focus on a better understanding of their pharmacokinetic properties, and on possible improvements through synthetic modifications.

\section{Competing interests}

The authors declare no competing interests.

\section{Authors' contributions}

HMM conceived the project. HMM, TW, MC, MOO, DH and PD performed the experiments. IN, SMS, AH, US, MH and RB supervised the work. All authors evaluated the results and revised the manuscript for publication. All the authors read and approved the final manuscript.

\section{Acknowledgements}

The German Academic Exchange Program (DAAD) for supporting this work by award of a scholarship grant A/03/44009 to H.M.M. The International Foundation for Science (IFS) through project grant F/3767-1 and Canton Basel Stipend Commission for the research visit grant to the Swiss Tropical and Public Health Institute (STPHI), the Institute of Organic Chemistry at UNIBAS and the Institute of Pharmaceutical Biology (PharmBio) at UNIBAS in Swizerland. Extraction and initial isolation of compounds was carried out at the Department of Traditional Medicine at the National Institute for Medical Research (NIMR) in Tanzania and the Department of Chemistry at Kenyatta University in Kenya. NIMR, the Institute of Organic Chemistry (UNIBAS), PharmBio (UNIBAS) and the STPHI are gratefully acknowledged for provision of laboratory space, supervison and financially supported this project.

\footnotetext{
Author details

${ }^{1}$ Department of Traditional Medicine Research, National Institute for Medical Research, P.O. Box 9653, Dar es Salaam, Tanzania. ${ }^{2}$ Medical Parasitology and Infection Biology, Parasite Chemotherapy Unit, Swiss Tropical Institute, University of Basel, Socinstrasse 57, CH-4002, Basel, Switzerland. ${ }^{3}$ Department of Chemistry, Kenyatta University, P.O. Box 43844, Nairobi, Kenya. ${ }^{4}$ International Centre for Insect Physiology and Ecology, P.O. Box 30772, Nairobi, Kenya. ${ }^{5}$ Institute of Organic Chemistry, University of Basel, St Johanns Ring 19, CH-4052, Basel, Switzerland. 'Institute of Pharmaceutical Biology, University of Basel, Klingelbergstrasse 50, CH-4056, Basel, Switzerland. ${ }^{7}$ Department of Pure and Applied Chemistry, Masinde Muliro University of Science \& Technology, P. O. Box 190, Kakamega, Kenya.
}

Received: 29 October 2012 Accepted: 4 February 2013

Published: 27 February 2013

\section{References}

1. WHO. The World Health Report: Reducing Risks, Promoting Healthy Life. Geneva: WHO; 2002.

2. WHO: Chemical Methods for Control of Arthropod Vectors and Pests of Public Health. Geneva, Switzerland: WHO; 1984

3. Marsden PD: American trypanosomiasis. In Manson's Tropical Diseases, 64. 20th edition. Edited by Cook G. New Delhi: W.B. Saunders \& Co; 1996:1197-1212.

4. Bryceson ADM: Leishmaniasis. In Manson's Tropical Diseases, 65. 20th edition. Edited by Cook G. New Delhi: W. B. Suanders \& Co; 1996:1213-1245.

5. Olliaro P, Cattani J: Malaria, the submerged disease. J Am Med Assoc 1996, 275:230.

6. Sandberg F, Cronlund A: What can we still learn from traditional folklore medicine? Examples from the results of a biological screening of medicinal plants from equatorial Africa, Volume 3. Colombo, Sri Lanka: Proc. 3rd Asian Symposium on Medicinal Plants and Spices; 1977:178-197.

7. Newman DJ, Cragg GM, Snader KM: The influence of natural products upon drug discovery. Nat Prod Rep 2000, 17:215-234.

8. Betti JL: Medicinal plants sold in Yaoundé markets, Cameroon. African Study Monographs 2002, 23:47-64.

9. Bouquet A, Debray M: Plantes médicinales de la Côte d'lvoire, Volume 32. Paris: Mémoires Office de la Recherche Scientifique et Technique d'OutreMer (O.R.S.T.O.M); 1974:232.

10. Wafo $P$, Nyasse B, Fontaine $C$, Sondengam BL: Aporphine alkaloids from Enantia chlorantha. Fitoterapia 1999, 70:157-160.

11. Atindehou KK, Schmid C, Brun R, Koné MW, Traore D: Anti-trypanosomal and anti-plasmodial activity of medicinal plants from Côote d'Ivoire. J Ethnopharmacol 2004, 90:221-227.

12. Agbaje EO, Onabanjo AO: The effects of extracts of Enantia chlorantha in malaria. Ann Trop Med Parasitol 1991, 85:585-590.

13. Leboeuf M, Cave A: Alcalöides des Annonacées. Plantes Médicinales et Phytothérapie 1972, 6:87-90.

14. Jössang A, Leboeuf M, Cave A: Alcalöides des Annonacées. XVII. Alcalöides de l'Enantia polycarpa Engl. et Diels. Planta Med 1977, 32:249-257.

15. Jössang A, Leboeuf M, Cave A: La polycarpine, nouvelle alcalöide isoquinolique de l'Enantia polycarpa Engler et Diels. Comptes Rendus de l'Académie des Sciences 1977, 284:567-569.

16. Buzas A, Osowiecki M, Regnier C: Sur la présence de quinidine (et I'hydroquinidine) dans l'écorce de l'Enantia polycarpa. Comptes Rendus Académie des Sciences 1959, 248:2791-2793.

17. Buzas $A$, Egnell $C$, Orsay F: Sur la présence de quinidine à côté d'alcaloïdes berberiniques dans les écorces d'Enantia pilosa et $E$. polycarpa. Annales Pharmaceutiques de France 1965, 23:351-354.

18. Vennerstrom JL, Klayman DL: Protoberberine alkaloids as antimalarials. J Med Chem 1988, 31:1087-1093.

19. Phillipson JD, Wright CW: Antiprotozoal agents from plant sources. Planta Med 1991, 57:53-59.

20. Virtanen P, Lassila V, Njimi T, Mengata DE: Natural protoberberine alkaloids from Enantia chlorantha, palmatine, columbamine and jatrorrhizine for thioacetamide-traumatized rat liver. Acta Anatomy (Basel) 1988, 131:166-170.

21. Guo Y, Kojima K, Lin L, Fu X, Zhao C, Hatano K, Chen Y-J, Ogihara Y: A new $\mathrm{N}$-methyltetrahydroprotoberberine alkaloids from Tinospora hainanensis. Chem Pharm Bull 1999, 47:287-289.

22. Trager $W$, Jenssen JB: Human malaria parasites in continuous culture. Science 1976, 193:673-675.

23. Desjardins RE, Canfield CJ, Haynes JD, Chulay JD: Quantitative assessment of antimalarial activity in vitro by a semi-automated microdilution technique. Antimicrob Agents Chemother 1979, 16:710-718.

24. Matile H, Pink JRL: Plasmodium falciparum malaria parasite cultures and their use in immunology. In Immunological Methods. Edited by Lefkovits I, Pernis B. Academic Press: San Diego; 1990:221-234.

25. Baltz T, Baltz D, Giroud C, Crockett J: Cultivation in a semi-defined medium of animal infective forms of Trypanosoma brucei, $\mathrm{T}$. equiperdum, T. evansi, T. rhodesiense and T. gambiense. EMBO J 1985, 4:1273-1277.

26. Räz B, Iten M, Grether-Bühler $Y$, Kaminsky R, Brun R: The Alamar Blue assay to determine drug sensitivity of African trypanosomes ( $T$. b. rhodesiense and T. b. gambiense). Acta Trop 1997, 68:139-147. 
27. Ganapaty S, Thomas PS, Karagianis G, Waterman PG, Brun R: Antiprotozoal and cytotoxic naphthalene derivatives from Diospyros assimilis. Phytochem 2006, 67:1950-1956.

28. Tsai TH, Wang GJ, Lin LC: Vasorelaxing alkaloids and flavonoids from Cassytha filiformis. J Nat Prod 2008, 71:289-291.

29. Katsui N, Sato K: Alkaloids of Lysichton camtschatcense Schott var. Japonicum Makino. Tetrahedron Letters 1966, 50:6257-6261.

30. Chang Y-C, Chang FR, Wu Y-C: The constituents of Lindera glauca. J Chin Chem Soc 2000, 47:373-380.

31. Cortes D, Davoust D, Hadi AHA, Myint SH, Hoquemiller R, Cave A: Trivalvone, une nouvelle bis-aporphine des ecorces de Trivalvaria macrophylla. J Nat Prod 1990, 53:862-866.

32. Achenbach $\mathrm{H}$, Schwinn A: Aporphinoid alkaloids and terpenoids from Piptostigma fugax. Phytochem 1995, 38:1037-1048.

33. Halbsguth C, Meißner O, Häberlein H: Positive cooperation of protoberberine type 2 alkaloids from Corydalis cava on the $G_{A B A_{A}}$ binding site. Planta Med 2003, 69:305-309.

34. Li C-Y, Lu H-J, Lin C-H, Wu T-S: A rapid and simple determination of protoberberine alkaloids in cortex phellodendri by ${ }^{1} \mathrm{H}$ NMR and its application for quality control of commercial traditional Chinese medicine prescriptions. J Pharm Biomed Anal 2006, 40:173-178

35. Grycová L, Dostál J, Marek R: Review: Quaternary protoberberine alkaloids. Phytochemistry 2007, 68:150-175.

36. Malebo HM, Tanja W, Cal M, Swaleh SM, Omolo MO, Hassanali A, Séquin U, Hamburger M, Ndiege IO, Brun R: Anti-plasmodial, anti-trypanosomal, anti-leishmanial and cytotoxicity activity of selected Tanzanian medicinal plants. Tanzania J Heal Res 2009, 11(4):226-234.

37. Muhie S: Chemical Investigations for Anti-malarial and Anti-trypanosomal and other Chemical Constituents of Uvaria lucida Lucida and Enantia kummeriae. M.Sc. Thesis. Tanzania: University of Dar-es-Salaam; 1996.

38. Rasoanaivo P, Ratsimamanga-Urverg S, Rakoto-Ratsimamanga A, Raharisololalao A: Constituants chimiques de trois especes de Burasaia (Menispermacees) endemiques de Madagascar. Biochem Syst Ecol 1991, 19:433-437

39. Hsieh TJ, Chia YC, Wu YC, Chen CY: Chemical constituents from the stems of Mahonia japonica. J Chin Chem Soc 2004, 51:443-446.

40. Hussain RA, Kim J, Beecher CWW, Kinghorn D: Unambiguous carbon-13 NMR assignments of some biologically active protoberberine alkaloids. Heterocycles 1989, 29:2257-2260.

41. Blanchfield JT, Sands DPA, Kennard CHL, Karl A, Byriel KA, Kitching W: Characterisation of alkaloids from some Australian Stephania (Menispermaceae) species. Phytochem 2003, 63:711-720.

42. Iwasa K, Nishiyama Y, Ichimaru M, Moriyasu M, HyeSook K, Wataya Y, Yamori T, Takashi T, Lee D: Structure-activity relationships of quaternary protoberberine alkaloids having an antimalarial activity. Eur J Med Chem 1999, 34:1077-1083.

doi:10.1186/1472-6882-13-48

Cite this article as: Malebo et al: Anti-protozoal activity of aporphine and protoberberine alkaloids from Annickia kummeriae (Engl. \& Diels) Setten \& Maas (Annonaceae). BMC Complementary and Alternative Medicine 2013 13:48.

\section{Submit your next manuscript to BioMed Central and take full advantage of:}

- Convenient online submission

- Thorough peer review

- No space constraints or color figure charges

- Immediate publication on acceptance

- Inclusion in PubMed, CAS, Scopus and Google Scholar

- Research which is freely available for redistribution 